\title{
Temporal variability of euphausiid concentrations in a Nova Scotia shelf basin using a bottom- mounted acoustic Doppler current profiler
}

\author{
N. A. Cochrane', D. D. Sameoto ${ }^{2}$, D. J. Belliveau ${ }^{1}$ \\ ${ }^{1}$ Physical and Chemical Sciences, Department of Fisheries and Oceans, Bedford Institute of Oceanography, Dartmouth, \\ Nova Scotia, Canada B2Y 4A2 \\ ${ }^{2}$ Biological Sciences, Department of Fisheries and Oceans, Bedford Institute of Oceanography, Dartmouth, Nova Scotia, \\ Canada B2Y 4A2
}

\begin{abstract}
The usefulness of bottom-mounted, upward-looking sonars for long-term quantitative zooplankton monitoring was demonstrated utilizing a $49 \mathrm{~d}$ record of acoustic volume backscattering strengths collected by a $150 \mathrm{kHz}$ RDI Acoustic Doppler Current Profiler in LaHave Basin off Nova Scotia, Canada. Variations in the character and intensity of 2 distinct acoustic scattering horizons distinguished by differing diurnal migration patterns were noted. One horizon confined to the top $50 \mathrm{~m}$ and only present during the early parts of the recording was tentatively ascribed to juvenile fish. Another strongly migrating horizon identified with the euphausiid Meganyctiphanes norvegica was studied in detail. $M$. norvegica in daytime were broadly distributed at about $150 \mathrm{~m}$ depth. At night they were uniformly distributed in the upper $50 \mathrm{~m}$. A $30 \%$ decrease in nighttime integrated backscattering levels was consistent with euphausiid accumulation in the acoustically unobservable near-surface region. Average target strengths declined $\leq 1 \mathrm{~dB}$ during active vertical migration. This observation, interpreted by acoustic scattering theory, constrained average euphausid inclinations to little more than $30^{\circ}$ during upward swimming. Column integrated population densities were roughly $290 \mathrm{~m}^{-2}$ Use of an inclined beam sonar geometry should result in backscattering levels less sensitive to organism orientation than use of a conventional vertically oriented sonar beam.
\end{abstract}

KEY WORDS: Sonar · Backscattering $\cdot$ Euphausiids $\cdot$ Acoustics

\section{INTRODUCTION}

A need exists to collect long time series of macrozooplankton and micronekton abundances. One promising methodology is the use of stationary bottom-mounted acoustic systems which have the ability to continuously record biological acoustic backscattering from virtually the entire water column and to monitor temporal changes in population concentrations and behaviours over an indefinite time period. On combining acoustic information with simultaneously collected physical and biological data it should be possible to determine the physical and biological events influencing major changes in populations of macrozooplankton such as euphausiids. With this purpose we examined the feasibility of utilizing an RD Instruments (RDI) Doppler sonar to monitor changes in scattering from euphausid concentrations and, with the assistance of acoustic scattering theory, to estimate population concentrations over an extended period. A more fundamental objective was to reduce the large variances in population estimates presently inferred from a limited number of discrete estimates collected over the short time span of our typical ship survey.

The inner Scotian Shelf basins contain a distinctive ecosystem supporting a large biomass of silver hake Merluccius bilinearis (Koeller et al. 1989), 3 deepdwelling (below $200 \mathrm{~m}$ ) Calanus species (Herman et. al. 1990, Sameoto \& Herman 1990), and the euphausiid Meganyctiphanes norvegica, a strong diurnal migrator (Cochrane et al. 1991). Previous ship-based acoustic studies (Cochrane et al. 1991, Sameoto et al. 1993) 
have demonstrated that, within Scotian Shelf basins, euphausiids are important and often the dominant acoustic scatterers in the frequency range 50 to $200 \mathrm{kHz}$. The $150 \mathrm{kHz}$ sonar used in the present survey was bottom-deployed on the northwestern slope of LaHave Basin immediately following a SeptemberOctober 1990 vessel acoustic survey in LaHave Basin (Sameoto et al. 1993). We will proceed assuming that, unless otherwise noted, the acoustic scattering signals originate primarily from euphausiids, an assumption which will be examined more critically in the 'Discussion' section below.

The use of RD Instrument Acoustic Doppler Current Profiler (ADCP) type sonars to gather amplitude, bioacoustic backscatter data is well established (Flagg \& Smith 1989, Thomson et al. 1989, Heywood et al. 1991, Roe \& Griffiths 1993). In recent years instrument performance in providing accurate amplitude data has been significantly improved. Surveys with bottommounted, internally recording sonars have 2 advantages over zooplankton surveys with vessel-mounted or towed sonars. First, and most obvious, long time series (up to 6 mo or more) are practical, allowing temporal variability to be studied. Secondly, mounting sonars solidly on the bottom ensures stable acoustic beam orientations. This permits the use of narrower beams than practical with unstabilized surface sonars without incurring the penalty of variable and inaccurate backscatter measurements at long range due to changes in beam orientation during signal transit time. Measurements with hull-mounted and towed sonars are plagued by trade-offs between quantitative accuracy, sea state and beam width. In addition, the inclined beam geometry of ADCP type sonars, in the common case of backscattering from elongate organisms with a roughly horizontal orientation, makes observed acoustic target strengths less critically dependent on target orientation compared to similar observations made with a vertically oriented transducer beam. This fact is demonstrated numerically below. The inclined beam geometry may therefore be advantageous for biological assessment if target orientations are poorly known or vary systematically over time.

To interpret an acoustic backscattering level as a quantitative abundance of animals an accurate organism target strength must be determined. Tractable theoretical formulations to precisely model target strengths of an organism as complex as a euphausiid are not available although significant progress has recently been made (Stanton et al. 1993a). Attempts to circumvent this problem by using empirical target strengths from natural in situ aggregate scattering, combined with population estimates from net sampling, are plagued by uncertainties due to net avoidance and small-scale inhomogeneous aggregation (Sameoto et al. 1993). Empirical target strengths from confined and controlled aggregations of organisms may not reflect the distribution of organism orientations and possibly other physical parameters characteristic of natural free-roving populations (Foote et al. 1990, Chu et al. 1993, Stanton et al. 1993a).

This study will describe and interpret ADCP backscatter amplitude data. Attention will be given to the spatial-temporal characteristics of the data, including the properties of the average diurnal vertical migratory cycle. An acoustic model incorporating both the unique ADCP beam geometry and a recently proposed semiempirical model for backscattering from fluid cylinders will be used to compute an euphausiid 'effective' target strength taking into account the best current estimates of their orientational behaviours. Using these target strengths and the observed backscattering strengths, quantitative estimates of euphausiid column density will be calculated.

\section{METHODS}

Acoustic instrumentation. Data were collected with an internally recording RD Instrument SC150 ADCP equipped with a nominal $150 \mathrm{kHz}, 20^{\circ}$ transducer head. The instrument was deployed on the northwestern flank of LaHave Basin in $192 \mathrm{~m}$ of water. The profiler was mounted in an enclosing, trawlproof package (Dessureault et al. 1991). The normal plastic transducer window was omitted enabling more precise quantitative measurement. Relevant instrument and recording parameters appear in Table 1. A Coastal Climate Minimet buoy moored nearby beyond the range of the acoustic beams recorded surface water temperatures and wind velocities to assist analysis.

Database. Just over $49 \mathrm{~d}$ of profile data were collected at $1 \mathrm{~h}$ sampling intervals. Each hourly backscattering value was obtained from the average of 10 min of acoustic sampling preceding the hour mark consisting of 1430 discrete acoustic pulses. Data were recorded for successive sampling bins of about $4.4 \mathrm{~m}$ vertical extent. The center of the first (deepest) bin was about $9 \mathrm{~m}$ above bottom. Bins centered shallower than $14 \mathrm{~m}$ were heavily contaminated by transducer side-lobe radiation scattered off the water surface and were not used for bio-acoustic assessment.

Data reduction. For quantitative analysis and display, raw amplitude counts from 1 selected beam were reduced to profiles of volume backscattering strength versus water-column depth using the parameters in Table 1 and the reduction procedure outlined by the manufacturer (RD Instruments 1990). This procedure 
Table 1. Instrument and recording parameters

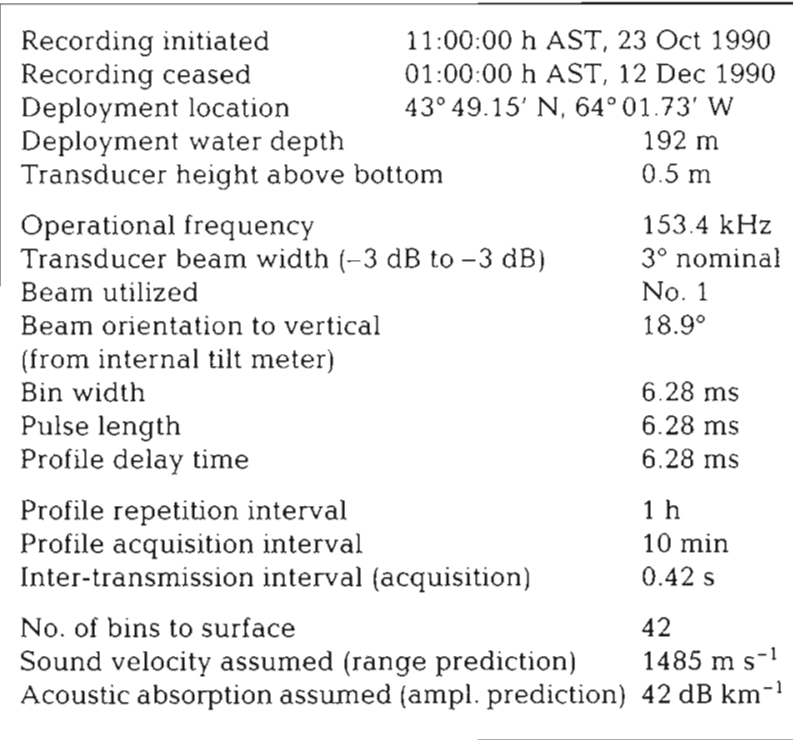

utilized instrument-specific calibrations supplied by the manufacturer, background reference levels established in post-cruise tank tests, and internal voltages and temperatures continuously recorded during deployment. Required acoustic absorption coefficients were computed using temperature and salinity data observed on 2 CTD casts at instrument deployment and 2 casts at recovery and the absorption relations of Francois \& Garrison (1982a, b). Column average acoustic attenuations decreased from 41.4 to $36.9 \mathrm{~dB}$ $\mathrm{km}^{-1}$ during the course of data collection. This was a consequence of progressive erosion of the upper $50 \mathrm{~m}$ seasonal mixed layer and significant changes in the deeper TS structures down to about $130 \mathrm{~m}$. An average attenuation of $39.1 \mathrm{~dB} \mathrm{~km}^{-1}$ was used for all data reductions.

Acoustic modeling. A realistic target strength is necessary to interpret backscattering levels in terms of scatterer abundance. A technique for computing an 'effective' target strength for elongate scatterers observed in an ADCP inclined beam is now outlined. For convenience, the general methodologies and notation of Cochrane et al. (1991) and Sameoto et al. (1993) are followed, these generally conforming to those of Clay \& Medwin (1977).

For a population of identical scatterers of target strength $T S(\mathrm{~dB})$ and concentration $N\left(\mathrm{~m}^{-3}\right)$ the volume backscattering strength $S_{\mathrm{v}}(\mathrm{dB})$ is given by:

$$
S_{\mathrm{v}}=T S+10 \log _{10} N
$$

If the scatterers are of identical size and geometry but differ in acoustic cross section because of their orientation to the beam, Eq. (1) may be retained by replacing $T S$ by an 'effective' target strength, $T S_{\text {eff, }}$ derived from the average backscattering cross section, $\bar{\sigma}_{\mathrm{bs}}$, of the ensemble:

$$
T S_{\text {eif }}=10 \log _{10} \bar{\sigma}_{\mathrm{bs}}
$$

Elongate organisms are modeled as straight, finite length, fluid cylinders with orientations described statistically by a probability distribution function (PDF) of known form. The orientational PDF, denoted $P_{2-D}(\theta)$, describes the probability of a cylinder axis being inclined at angle $\theta$ to the horizontal, for a sub-population of cylinders with axes lying close to a given vertical plane. In absence of definitive experimental data we approximate it by a Gaussian form:

$$
P_{2-\mathrm{D}}(\theta)=\frac{1}{\sigma(2 \pi)^{1 / 2}} e^{-\left\{\theta^{2} / 2 \sigma^{2}\right\}}
$$

It is assumed that the azimuthal distribution of cylinder orientations is random precluding choice of any preferred vertical plane. If one chooses to work with a 3-dimensional volumetric PDF rather than with a 2-dimensional planar PDF:

$$
P_{\text {3-D }}(\theta) \propto P_{2-\mathrm{D}}(\theta) \cos \theta
$$

In practice, the ADCP beam widths are much smaller than the range of aspect angles presented by the zooplankton. This permits one to omit consideration of the finite transducer beam widths thereby simplifying the computations (compare Sameoto et al. 1993). The average backscattering cross section is obtained as a probability-weighted ensemble average over all possible orientations of identical cylinders:

$$
\bar{\sigma}_{\mathrm{bs}}=\frac{\int_{0}^{\pi} \int_{-\pi / 2}^{\pi / 2} \sigma_{\mathrm{bs}}(\beta) P_{2-\mathrm{D}}(\theta) \cos \theta \mathrm{d} \theta \mathrm{d} \varphi}{\int_{0}^{\pi} \int_{-\pi / 2}^{\pi / 2} P_{2-\mathrm{D}}(\theta) \cos \theta \mathrm{d} \theta \mathrm{d} \varphi}
$$

where:

$$
\cos \beta=\sin \alpha \cos \varphi \cos \theta+\cos \alpha \sin \theta
$$

and $\alpha=$ transducer beam inclination from the vertical; $\theta=2$-dimensional (planar) dip angle of cylinder to horizontal; $\varphi=$ azimuthal orientation of cylinder axis.

The acoustic cross sections, $\sigma_{\mathrm{bs}}$, of individual ensemble members in Eq. (5) are functions of $\beta$, the orientation angle between the transducer acoustic axis and the cylinder axes (i.e. the acoustic grazing angle). For computation, acoustic cross sections have been obtained from 2 variations of the cylinder model, namely, the finite straight cylinder Ray model (Stanton et al. 1993b) and the corresponding high pass model (Stanton 1989). The newer Ray model incorporates a close approximation of modal resonances using geometric ray theory, while the older high pass model, utilized in our previous work, assumes individual modal resonances are smoothed to vanishing in an ensemble average when a degree of variability in ensemble 
Table 2. Finite straight cylinder scattering models of Stanton (1989) and Stanton et al. (1993b)

$$
\begin{aligned}
& \text { Cylinder Ray model } \\
& \qquad \sigma_{\mathrm{bs}}=\frac{L^{2}}{4 \pi} k_{1} a(\sin \beta) R_{\mathrm{t} 2}{ }^{2} s^{2}\left[1+\left(T_{12} T_{21}\right)^{2}-2 T_{12} T_{21} \cos \gamma\right] \\
& \text { where } \quad \gamma=4 k_{2} a \sin \beta-\frac{\pi}{2} \frac{k_{1} a}{k_{1} a+0.4} \\
& \text { Cylinder high pass model } \\
& \qquad \sigma_{\text {bs }}=\frac{1}{4} L^{2}(K a)^{4} \alpha_{\pi c}{ }^{2} s^{2} \\
& \text { where } \quad \alpha_{\pi c}=\frac{1-g h^{2}}{2 g h^{2}}+\frac{1-g}{1+g}
\end{aligned}
$$

member size and orientation are present. Relevant formulae appear in Table 2. Recent theoretical studies and laboratory measurements by Stanton et al. (1993a) strongly suggest the Ray model to be preferred over the high pass model at the acoustic wavelength and for the scatterer sizes characterizing this experiment.

Once an acoustic cross section has been computed from Eq. (5), scatterer concentrations can be calculated from measured volume backscattering strengths using Eqs. (1) \& (2).

\section{RESULTS}

A 3-dimensional echogram showing the volume backscattering coefficient, $s_{v}$, versus both watercolumn depth and time for the entire recording period is shown in Fig. 1. $S_{v}$ is the non-logarithmic form of $S_{v}$ i.e. $S_{v}=10 \log _{10} S_{v}$. Assuming a population of identical scatterers, Eq. (1) implies the vertical coordinate $s_{v}$ is proportional to the population density $N$. Several char- acteristics are immediately evident. First, diurnal migratory behaviour is evident throughout most of the recording. The major scattering horizons tend to lie either above $50 \mathrm{~m}$ depth or below $110 \mathrm{~m}$. Transitions between these 2 configurations are comparatively rapid. Secondly, during approximately the first $500 \mathrm{~h}$ of recording a comparatively intense scattering horizon appears in the upper $50 \mathrm{~m}$ while little or no corresponding increase in scattering occurs below $50 \mathrm{~m}$, i.e. the intense scattering horizon remains confined to the upper water column at all times. Intensities in this horizon peak at depths of 30 to $40 \mathrm{~m}$ during the day. At night the zone of maximum intensity weakens, broadens considerably, and is centered nearer the surface suggestive of a limited upward migration. Thirdly, beyond Hour 500 there is a much stronger visual correlation between abundances observed in the upper and lower halves of the water column. Virtually the entire population shows a strong diurnal tendency. A few sharp spikes in acoustic intensity occur especially near Hours $350,465,490$ and 815 . These appear to be the lower remnants of scattering horizons extending down from the unobservable near-surface region. Since these events were only observed during periods of intense winds recorded by the Minimet buoy and since their acoustic intensities decline very rapidly with depth they are very likely bubble plumes (Thorpe 1992). Finally, systematic changes in total column scattering strength appear to occur over time intervals of several days. Irregularity is also noted in the strength of the diurnal migrations, i.e. during some time intervals the scatterers have a tendency to be distributed over a broader depth range or spend more time at mid-water column depths rather than the otherwise rapid alternation between a near-surface or near-bottom distribution. Examples occur in the vicinity of Hours 600 and 1020.

\section{ANALYSIS}

\section{Recorded data}

To illustrate the character of the records in more detail plots of column integrated $s_{v}$ versus time for the depth ranges 14 to $185 \mathrm{~m}$ (lower bound of lowermost bin), 14 to $90 \mathrm{~m}$, and 90 to $185 \mathrm{~m}$ for the complete recording period are shown in Fig. 2. The diurnal variation is clearest in the 90 to $185 \mathrm{~m}$ plot as fairly regular periodic pulses of sharply enhanced backscattering of roughly 8 to $9 \mathrm{~h}$ duration. In the 14 to $90 \mathrm{~m}$ plot, periodic depressions occur in integrated $s_{\mathrm{v}}$ at points corresponding to the periodic peaks in the 90 to $185 \mathrm{~m}$ plot. When the upper and lower water-column integrations are combined so as to extend over the entire 14 to 


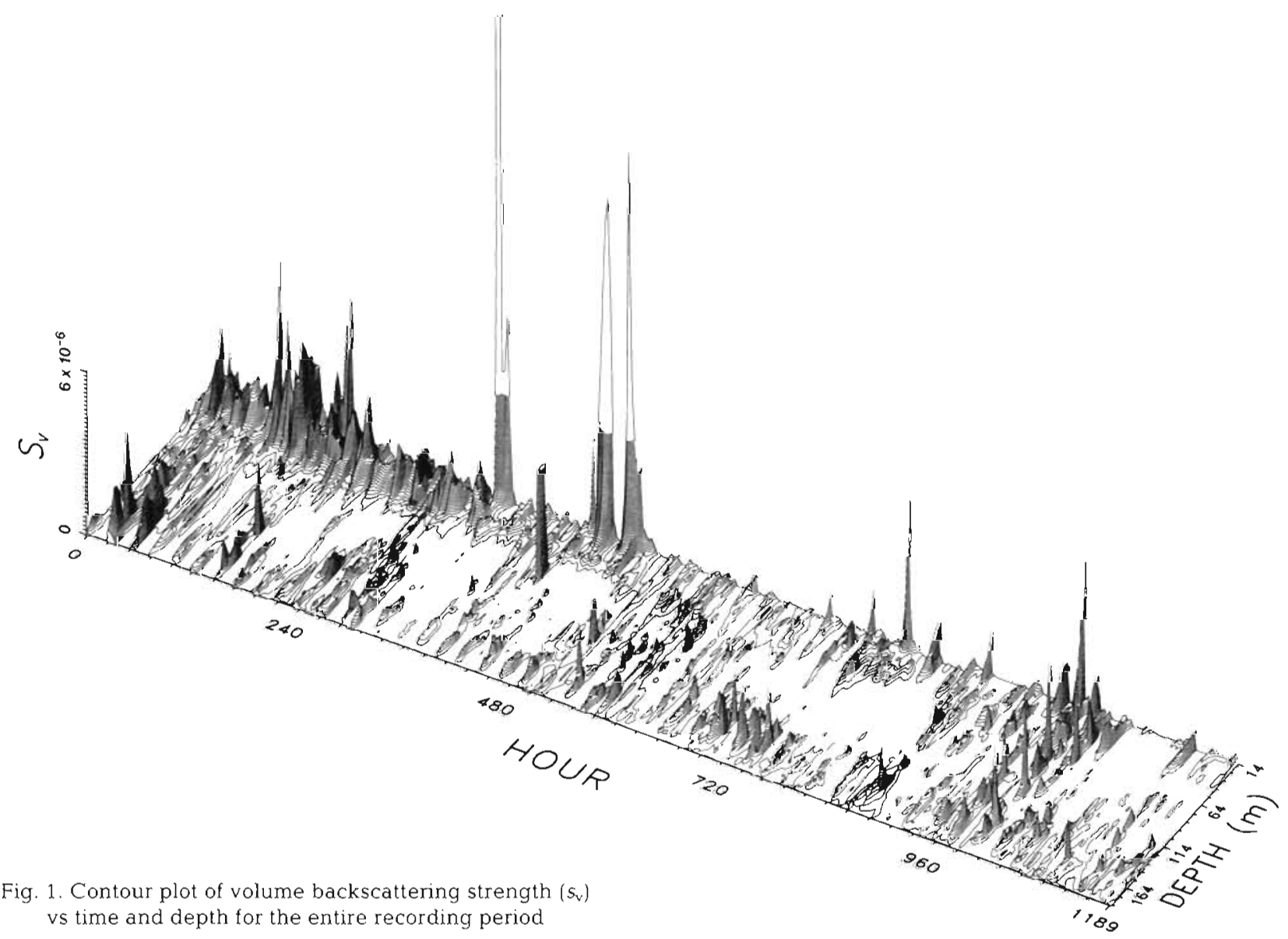

$185 \mathrm{~m}$ profiled range, the diurnal fluctuations largely disappear. Also evident on all 3 plots are narrow spikes lasting from $1 \mathrm{~h}$ or less (i.e. unresolved) to several hours corresponding to the passage of spatially localized intensely scattering aggregations of unknown nature. Longer period ( $>24$ h) trends are also evident, most clearly seen in the 14 to $185 \mathrm{~m}$ plot.

The corresponding column-integrated $s_{\mathrm{v}}$ values are shown in Fig. 3 for the same recording period after the diurnal effect has been suppressed by application of a symmetrical 25 point running mean filter. Several effects suggested in Fig. 2 are clarified in Fig. 3. First the long-term trend in total water-column integrated levels appears more closely related to upper rather than lower water-column populations. In particular, the large upper water-column population between Hours 100 to 500, which dominates the columnintegrated levels, shows little tendency to diurnally migrate into the lower water column. As noted earlier this shallow population does undergo a limited range diurnal migration confined to the top $50 \mathrm{~m}$. For the remainder of the recording, the smoothed lower watercolumn integrated backscattering levels tend to be comparable to or slightly lower than those in the upper water column. It should be remembered that each smoothed integrated level is dependent upon the total time the population spends within the relevant depth range as well as the equilibrium concentration; the day-night duration asymmetry implies less time on bottom than on top. During several brief periods, the lower water-column concentrations are less than those in the upper water column. The most evident example lies between Hours 1050 and 1125 where Fig. 1 indicates virtually no diurnal migration above $90 \mathrm{~m}$.

To investigate the nature of the mean diurnal variation, we have performed a $24 \mathrm{~h}$ stacked average of total water-column integrated $s_{v}$ levels between Hours 505 to 840 inclusive, while eliminating the heavily noise-contaminated bins above $14 \mathrm{~m}$ (Fig. 4). This window period was selected for 2 reasons. First, the diurnal variation appeared fairly regular (Fig. 1) without either a persistent surface layer (as before Hour 500) or obvious spurious signals from bubble clouds (the isolated event near point 815 only affects raw data above $14 \mathrm{~m}$ but is visible in Fig. 1 because of the slight vertical smoothing introduced by the contouring display algorithm). Secondly, the short $14 \mathrm{~d}$ time window should effectively nullify any effect of changing length of day on the timing of the 

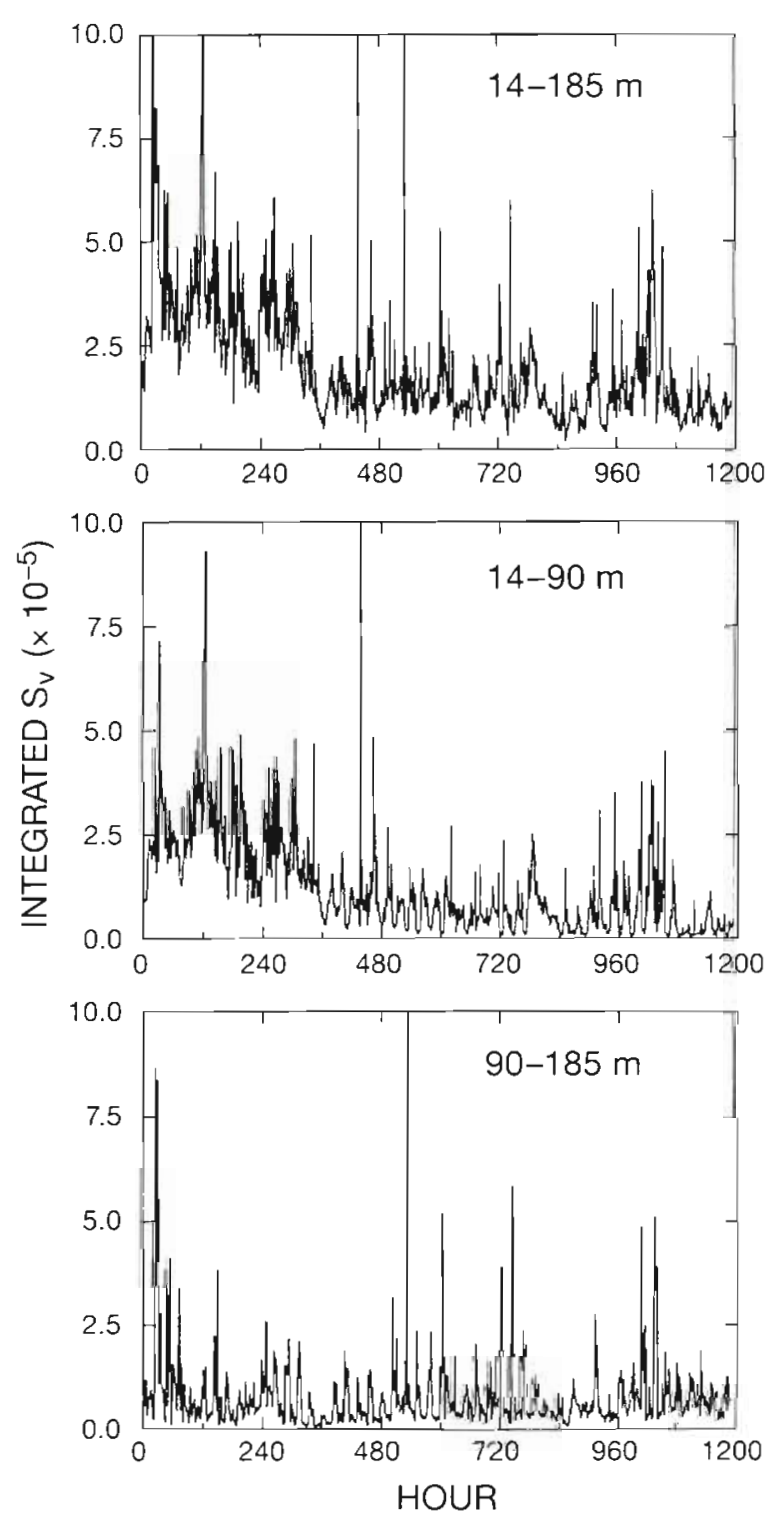

Fig. 2. Plots of depth integrated $\mathrm{s}$, vs time extending through the complete recording period. The depth range 14 to $185 \mathrm{~m}$ encompasses the entire profiled water column, while depth sub-ranges 14 to $90 \mathrm{~m}$ and 90 to $185 \mathrm{~m}$ correspond to the upper and lower portions of the profiled water column

diurnal migration, assuming of course that the diurnal cycle is linked to the solar cycle. Astronomical calculations using the techniques outlined by Meeus (1988) show that the times of local sunrise and sunset vary by 61 . and 41 min respectively over the complete $49 \mathrm{~d}$ recording interval compared to only 18 and 11 min respectively for the chosen window. Note that variations in times of sunrise and sunset over the window period are small compared to the 1 h sampling interval so that each hourly mean represents essentially an identical solar elevation or light level disregarding atmospheric effects. The mean times for sunrise and sunset are marked on Fig. 4.
The average diurnal pattern consists of 2 relatively stable scatterer configurations, namely a lower watercolumn scattering horizon during the day and an upper water horizon during the night (Fig. 4). The transitions between configurations are brief, occupying little more than the $1 \mathrm{~h}$ sampling interval. Upward migration occurs largely between 16:00 and 17:00 h AST (Atlantic Standard Time) and downward migration largely between 07:00 and 08:00 h AST. These times are close to visual sunrise and sunset when light levels change rapidly.
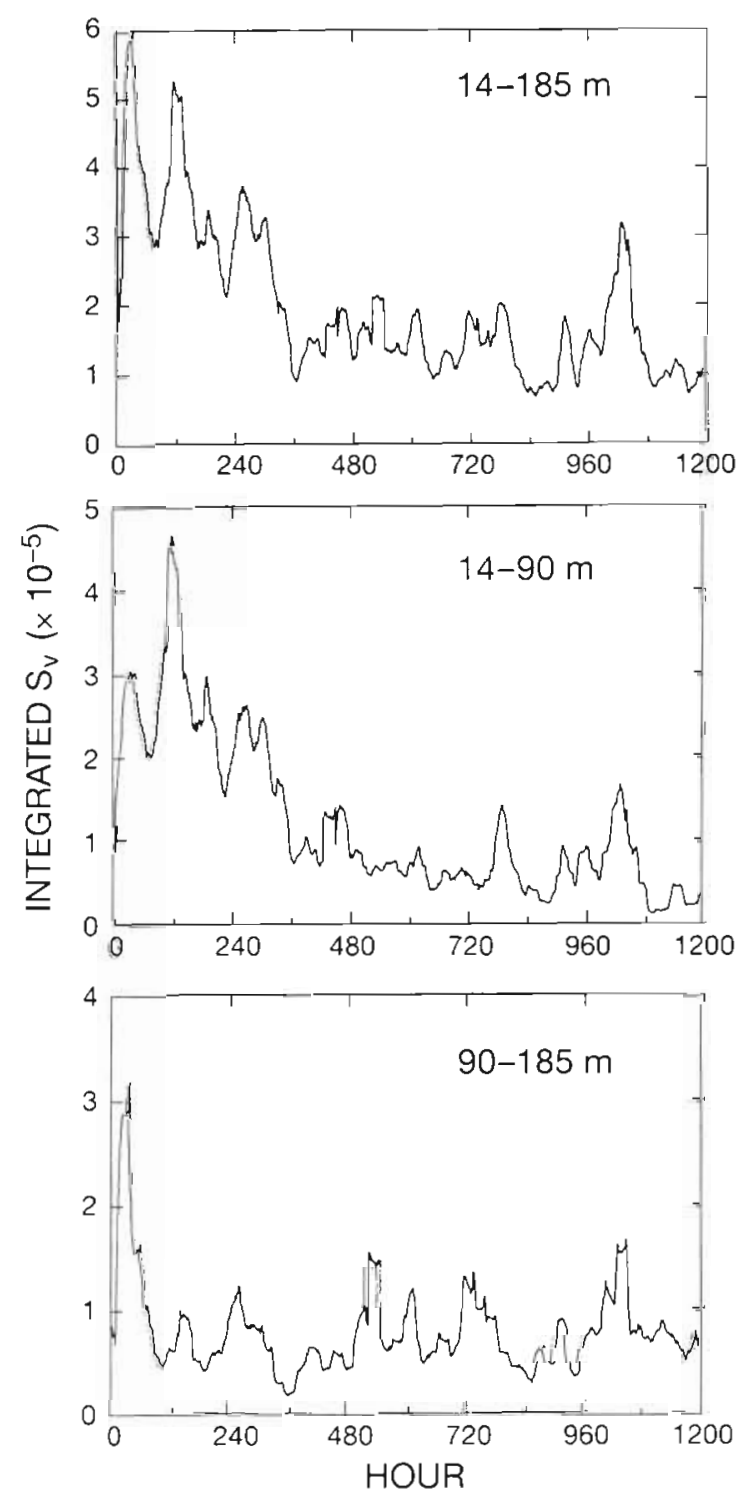

Fig. 3. Plots of smoothed integrated $s_{v}$ vs time extending through the complete recording period. The depth range 14 to $185 \mathrm{~m}$ encompasses the entire profiled water column, while depth sub-ranges 14 to $90 \mathrm{~m}$ and 90 to $185 \mathrm{~m}$ correspond to the upper and lower portions of the profiled water column. Smoothing was accomplished by applying a $25 \mathrm{~h}$ running mean over the data. Individual plots have been rescaled to emphasize detail 


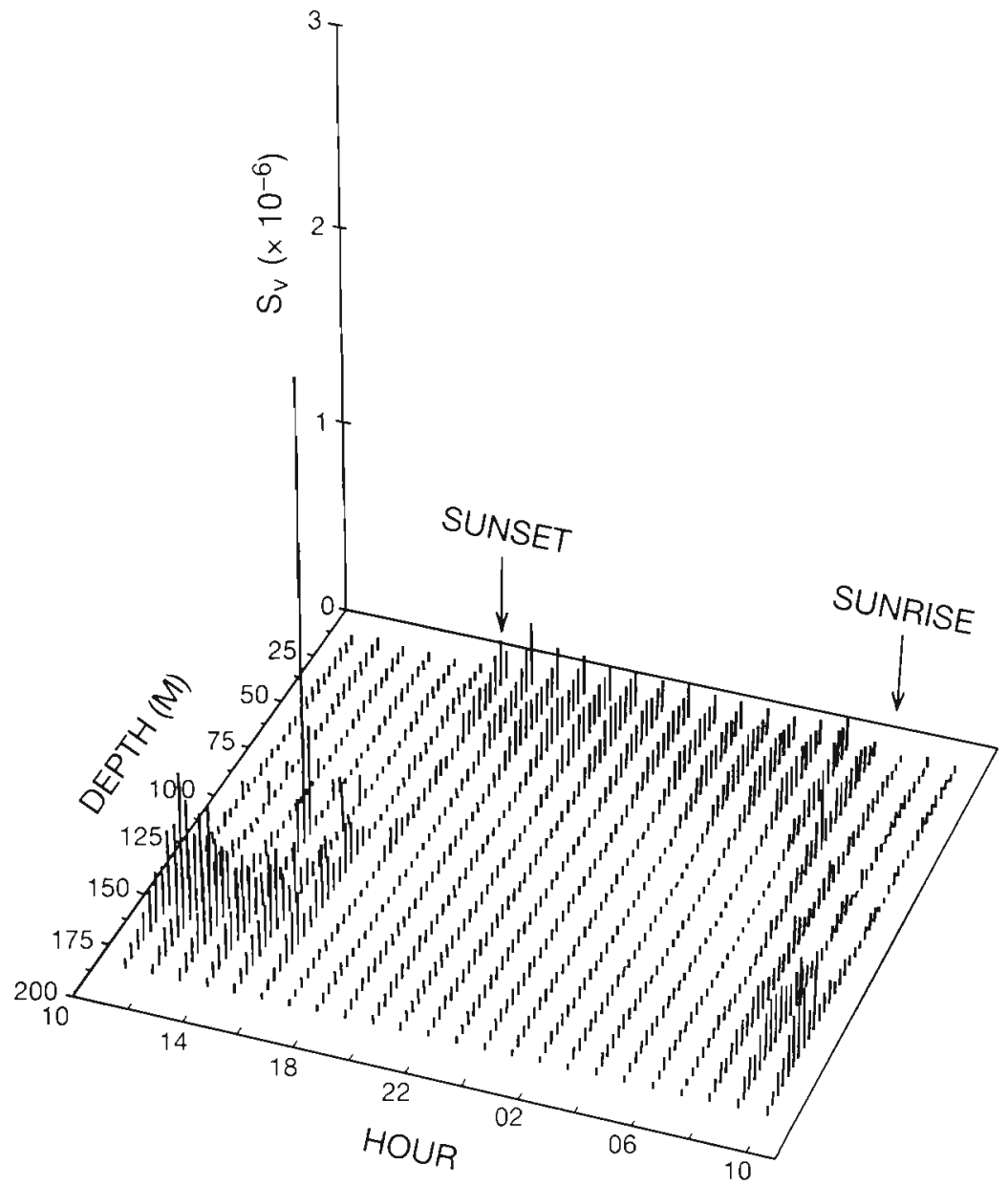

Fig. 4. Stick plot of averaged hourly $s_{v}$ vs depth and time of day over Hours 505 to 840 inclusive without smoothing

Fig. 5 shows the average hourly, column-integrated $s_{\mathrm{v}}$ for the stacked section of Fig. 4 with standard error bars except that the spike in $s_{\mathrm{v}}$ at 14:00 $\mathrm{h}$ in the stacked section (Fig. 4), traced to 1 anomalously high data point on a single day of recording, was suppressed. In general, integrated backscattering levels are about 30\% lower with the scattering horizon near-surface than when it is deep. The most natural explanation is that a fraction of the scatterers rise into the top $14 \mathrm{~m}$ of water column which is excluded from the integration. (Note that an analogous situation appears not to occur with the layer near bottom as the layer scattering strength decreases rapidly near its base with virtually the entire layer remaining above the $7 \mathrm{~m}$ gap between the lower most recorded bin and the physical bottom. Of course, in the absence of near-bottom data this cannot be proven absolutely.) If one considers the euphausiid layer to occupy the top $50 \mathrm{~m}$ with a uniform average concentration over this depth range, an assumption reasonably consistent with Fig. 4 as well as previous sampling (Sameoto et al. 1993) with an opening and closing net sampler, the
BIONESS (Sameoto et al. 1980), about $28 \%$ of the population would be excluded from the acoustic sum. This would be consistent with the reduction in integrated levels noted above.

At this point one should also consider whether inaccuracies in correcting for the approximate $280 \mathrm{~m}$ differential slant path absorption (from center of shallow distribution to center of deep distribution) or inaccuracies inherent in the sonar electronics could contribute significantly to the observed reduction in integrated levels. Using the temperature and salinity profiles at the beginning and at the end of the deployment period, the 2-way path absorptions would differ by about $1.3 \mathrm{~dB}$. The average of the 2 path absorptions was used to compute the data displayed in Fig. 5. Minimet buoy recordings showed that at the time of the stacked section surface temperatures had decreased to values comparable to those at the end of the deployment. If the deeper temperature-salinity structures had also readjusted (unknown) so that the acoustic path absorptions were comparable to those at the end of the deployment, $s_{\mathrm{v}}$ levels with biology near surface would be overestimated by about $0.6 \mathrm{~dB}$, i.e. 15 to $16 \%$, using our 'mean' attenuation approximation. It can be seen that any error in the assumed absorption coefficient is likely to be in the direction to further increase the excess of daytime integrated $s_{\mathrm{v}}$ over corresponding nighttime values. In addition, the basic uncertainty in computing the absorption for any one

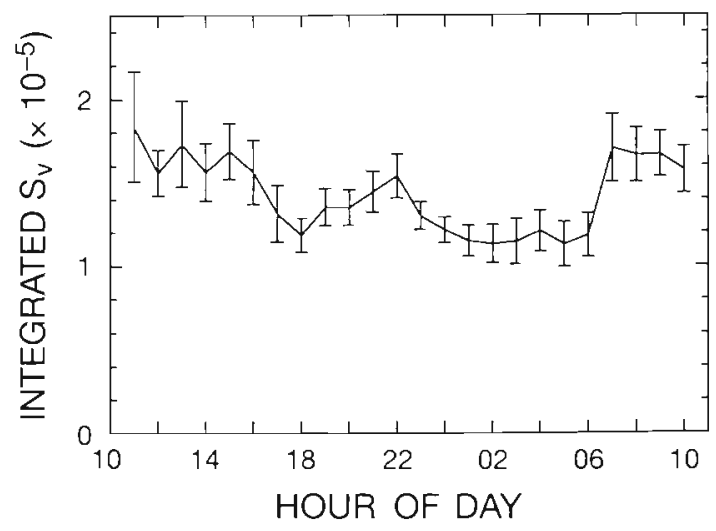

Fig. 5. Plot of hourly averaged total water column integrated $s_{v}( \pm \mathrm{SE})$ over Hours 505 to 840 inclusive 
structure using the Francois \& Garrison $(1982 \mathrm{a}$, b) formulation is of the order of $0.3 \mathrm{~dB}$ at our target range. $\mathrm{A}$ careful analysis of error inherent in the sonar's signal amplitude measurement capability has been performed by the manufacturer (RD Instruments 1990). For the range of signal levels observed, we estimate the differential - as opposed to absolute - uncertainty in integrated $s_{\mathrm{v}}$ (Fig. 5) is about $10 \%$ or $0.4 \mathrm{~dB}$ which is about equal to the measurement quantization interval. In the worst possible case, assuming a given temperaturesalinity structure, the total uncertainty in differential path absorption (biology near surface vs biology deep) is almost certainly less than $1 \mathrm{~dB}$ or $26 \%$ in day versus night integrated $s_{v}$ levels. Any additional bias introduced by our assumption of a specific temperaturesalinity structure, as noted above, has probably underestimated the true day to night differential. A final possibility is a change in integrated $s_{\mathrm{v}}$ due to differing effective target strengths of organisms near surface than when deep. These could arise from systematic changes in orientational behaviour.

We presently believe biological accumulation in the upper $14 \mathrm{~m}$ to be the simplest and most natural explanation for the reduction in integrated backscattering levels when the scatterers are near surface. Since this can quantitatively account for the entire observed effect, the absorption corrections and instrument calibrations used are probably more accurate than the worst possible case. This conclusion is reinforced by the observation of a marked jump in scattering levels between 06:00 and 07:00 $\mathrm{h}$ at the time when Fig. 4 indicates organisms have just fallen out of the top $14 \mathrm{~m}$ at the start of the downward migration. The slight reduction in integrated $s_{\mathrm{v}}$, compared to the lower configuration equilibrium values, observed in the period $16: 00$ to $17: 00 \mathrm{~h}$ during the upward migration may be real but, if so, does not exceed $1 \mathrm{~dB}$. No analogous drop is observed in the period 06:00 to 07:00 h during the downward migration. The tight constraints on effective target strengths during vertical migration will be seen to place limits on euphausiid orientations when interpreted by acoustic scattering theory.

\section{Quantitative modeling}

Table 3 lists computed euphausiid target strengths at $153.4 \mathrm{kHz}$ as functions of both the acoustic beam inclination to the vertical and the standard deviation of organism orientations about an assumed mean horizontal orientation. The target strengths tabulated were calculated using the straight cylinder Ray scattering model of Stanton et al. (1993b) above. The corresponding target strengths were also computed using the
Table 3. Straight cylinder Stanton Ray model. Theoretical effective target strengths $(\mathrm{dB})$ of a $0.028 \mathrm{~m}$ length euphausiid ensonified at $153.4 \mathrm{kHz}$ with a narrow acoustic beam of tabulated inclination to the vertical. Euphausiid target strengths are modeled utilizing an ensemble of fluid cylinders $(g=1.05$, $h=1.03$ ) with a planar distribution of orientation angles about the horizontal of Gaussian form and of tabulated width. The 'random' case refers to no preferential distribution of the ensemble (cylinder) axes vectors projected onto the surface of an enclosing sphere

\begin{tabular}{|ccccccc|}
\hline \multirow{2}{*}{$\begin{array}{l}\text { Beam } \\
\text { inclination }\end{array}$} & $0^{\circ}$ & $5^{\circ}$ & $10^{\circ}$ & $20^{\circ}$ & $30^{\circ}$ & Random \\
\hline $0^{\circ}$ & -63.4 & -65.7 & -67.9 & -70.5 & -71.8 & -74.2 \\
$15^{\circ}$ & -70.1 & -69.8 & -69.7 & -71.0 & -72.1 & -74.2 \\
$20^{\circ}$ & -71.4 & -71.2 & -70.8 & -71.4 & -72.3 & -74.2 \\
$30^{\circ}$ & -73.0 & -72.9 & -72.7 & -72.4 & -72.7 & -74.2 \\
$90^{\circ}$ & -76.1 & -76.1 & -76.0 & -75.8 & -75.5 & -74.2 \\
\hline
\end{tabular}

Stanton (1989) high pass straight cylinder model as employed in Sameoto et al. (1993). In both computations we utilized a 2-dimensional (planar) Gaussian PDF of organism inclination angles about the horizontal as outlined above. [To ease conceptualization we assume a distinction between organism head and tail and perform the integration in $\theta$ (Eq. 5) over the range $-\pi / 2$ to $+\pi / 2$ although the cylinder models employed in our simulations do not possess head-tail asymmetry.] Euphausiid physical parameters are identical to those used by Sameoto et al. (1993), namely: length $(L)=$ $0.028 \mathrm{~m}$, with cylindrical radius (a) assigned so as to preserve total organism volume, and $g=1.05$ and $h=1.03$ for $g$ and $h$ as defined in Table 2. The relationship between $a$ and $L_{r}$ in MKS units, computed using the equivalent spherical volume radius to organism length regression relations of Greenlaw \& Johnson (1982), is:

$$
a=0.0289 L^{0.79}
$$

Ray model target strengths consistently exceed the high pass target strengths by approximately $6.6 \mathrm{~dB}$. This arises because the $k a$ value of 1.10 (Table 2), corresponding to the ensonification frequency of $153.4 \mathrm{kHz}$ and target size above, lies close to the first resonant peak in reduced target strength (Stanton et al. 1993b); this peak is strongly smoothed in the high pass approximation. The specific $k a$ value also results in target strengths relatively insensitive to organism size. A sample computation, averaging backscattering cross sections over a realistic ensemble of organism sizes consistent with those for Scotian Shelf basin euphausiids (Sameoto et al. 1993), resulted in an effective target strength within $0.1 \mathrm{~dB}$ of that computed using the average length organism. Accordingly we have utilized only average lengths in our computations. 
Examination of Table 3 shows that target strengths measured with an inclined acoustic beam are generally less sensitive to the degree of scatter in organism inclinations (about the horizontal) than corresponding target strengths measured with a vertically directed beam. For example, using a $20^{\circ}$ inclined beam, target strengths vary by only $1.5 \mathrm{~dB}$ between (organism) inclination SDs of $10^{\circ}$ and $30^{\circ}$ compared to a variability of $3.9 \mathrm{~dB}$ using a vertically directed beam. Target strengths appear to maximize at an orientational SD magnitude slightly less than the beam inclination angle, but the effect is not marked. For a horizontal acoustic beam (inclination $90^{\circ}$ ), the effects of organism inclinations about the horizontal are not completely negated, a result perhaps contrary to initial intuitive expectations.

The cases presented in Table 3 assume organism tilts distributed symmetrically about a mean horizontal inclination. The effect of a non-horizontal mean tilt is shown in Fig. 4 using our acoustic beam inclination of $20^{\circ}$. For directive acoustic scatterers, such as euphausiids, the effect of a non-horizontal mean inclination is most pronounced when few ensemble members approach broadside incidence to the acoustic beam. This condition is fulfilled when the organism mean inclination to the horizontal exceeds the beam inclination to the vertical by about $1 \mathrm{SD}$ in the range of organism tilts. Because target strengths measured with an inclined acoustic beam are less sensitive to mean organism tilts deviating from the horizontal, the inclined beam geometry would appear advantageous for quantitative assessment of euphausiid populations where scatterer mean inclinations are uncertain - the normal case for present-day euphausiid studies - or suspected to vary with time such as during a vertical migratory cycle.

As noted above, the drop in water-column integrated $s_{v}$ levels when the euphausiids are in rapid upward migration appears $\leq 1 \mathrm{~dB}$. There is no evidence of a similar drop during downward migration. The model derived target strengths presented in Table 4 suggest that mean tilts do not greatly exceed $30^{\circ}$ during upward migration provided the average tilt during the stable periods (not migrating) is $\leq 15^{\circ}$ and assuming a fairly high degree of statistical scatter (e.g. $30^{\circ} \mathrm{SD}$ ) is also present. These constraints on orientation will be examined in the next section in the light of what is known or can be readily inferred about euphausiid behaviours.

Euphausiid column densities can be roughly estimated assuming a population of identical organisms. Our RDI profiler was calibrated by the manufacturer to an absolute uncertainty of about $2 \mathrm{~dB}$. Taking column integrated $s_{\mathrm{v}}$ of about $1.7 \times 10^{-5}$ for the section displayed in Fig. 5 and an 'effective' single euphausiid
Table 4 . Straight cylinder Stanton Ray model $\left(20^{\circ}\right.$ beam inclination). Theoretical effective target strengths $(\mathrm{dB})$ of a $0.028 \mathrm{~m}$ length euphausiid ensonified at $153.4 \mathrm{kHz}$ with a narrow acoustic beam inclined $20^{\circ}$ to the vertical. Euphausiid target strengths are modeled utilizing an ensemble of fluid cylinders ( $g=1.05, h=1.03)$ with a planar distribution function of orientation angles about a tabulated mean orientation to the horizon. The angular distribution function is assumed of Gaussian form and tabulated width

\begin{tabular}{|ccccccc|}
\hline $\begin{array}{l}\text { Average } \\
\text { orientation } \\
\text { angle }\end{array}$ & $0^{\circ}$ & $5^{\circ}$ & $10^{\circ}$ & $20^{\circ}$ & $30^{\circ}$ & Random \\
\hline $0^{\circ}$ & -71.4 & -71.2 & -70.8 & -71.4 & -72.3 & -74.2 \\
$15^{\circ}$ & -69.3 & -70.1 & -71.1 & -71.9 & -72.5 & -74.2 \\
$20^{\circ}$ & -69.8 & -70.8 & -71.7 & -72.4 & -72.7 & -74.2 \\
$30^{\circ}$ & -84.0 & -78.0 & -74.8 & -73.6 & -73.3 & -74.2 \\
$45^{\circ}$ & -87.7 & -88.3 & -83.7 & -76.6 & -74.7 & -74.2 \\
$60^{\circ}$ & -95.0 & -93.7 & -91.7 & -80.9 & -76.5 & -74.2 \\
$90^{\circ}$ & -109.2 & -108.6 & -101.7 & -91.2 & -81.2 & -74.2 \\
\hline
\end{tabular}

target strength of about $-72.3 \mathrm{~dB}$ (Table 4) Eq. (1) implies an average integrated column density of $290 \mathrm{~m}^{-2}$ (range of uncertainty: 180 to $460 \mathrm{~m}^{-2}$ ) corresponding to an average volume density (concentration) of $1.7 \mathrm{~m}^{-3}$. The highest average $s_{\mathrm{v}}$ levels in the lower water column in Fig. 4 are of the order of $6 \times 10^{-7}$ (11:00 $\mathrm{h}$ at about $140 \mathrm{~m}$ depth) corresponding to concentrations of about $10 \mathrm{~m}^{-3}$.

\section{DISCUSSION}

The pattern of diurnal euphausiid migration above is consistent with that outlined for the Scotian Shelf basins from more limited ship observations (Cochrane et al. 1991). Vertical migration velocities on the stacked section appear to equal or exceed $100 \mathrm{~m}$ over $1 \mathrm{~h}$, or $0.028 \mathrm{~m} \mathrm{~s}^{-1}$, compared to a vertical velocity of $0.04 \mathrm{~m} \mathrm{~s}^{-1}$ for the shallower, clearly discerned portion of the euphausiid migration reported in Cochrane et al. (1991). It is unlikely that the diurnal migration of silver hake previously studied in the Shelf basins (Cochrane et al. 1991) is implicated in these observations. Adult silver hake tend to be tightly confined to the deeper portions of the basins and should be scarce or absent at the bathymetric contour the RDI profiler was deployed. In addition, adult silver hake tend to spread out through a considerable portion of the water column at night with peak concentrations at or below the base of the $70 \mathrm{~m}$ thick seasonal mixed layer. This contrasts with the present almost exclusive upper $50 \mathrm{~m}$ nighttime distribution of acoustic scatterers but conforms quite closely to the distribution of euphausiids inferred from previous BIONESS sampling (Sameoto et al. 1993). 
The fact that daytime and nighttime column-integrated backscattering levels are comparable (within approximately $30 \%$ ), including levels during the periods of vertical readjustment, strongly suggests the bulk of the population remains suspended in the water column above the profiler when the population is deep (daytime) rather than accumulating directly on the bottom. Sampling with the BIONESS $1 \mathrm{~m}$ above bottom and also through the water column during both day and night, plus video observations of the bottom conducted in June 1990, showed few euphausiids close to or on the bottom in the day and even fewer at night. Ship observations in the center of LaHave Basin (Cochrane et al. 1991) and a combined net sampling, acoustics, and optical counter survey in LaHave Basin between 28 and 30 September 1990 (Herman et al. 1993), just prior to the present study, showed Meganyctiphanes norvegica present at the depth of the RDI mooring undergoing diurnal migrations. These data showed that the maximum of the daytime euphausiid layer $(220 \mathrm{~m})$ lies below the RDI deployment depth $(192 \mathrm{~m})$ but maximizes at a comparable height $(20$ to $30 \mathrm{~m})$ above bottom. This indicates the daytime layer depth is more strongly influenced by the proximity of the bottom rather than by ambient light level or other depthdependent criteria. Bottom avoidance could represent a behavioural adaptation to near-bottom dwelling predators. Little systematic vertical migratory behaviour can be discerned during the hours of total darkness. This observation is consistent with the laboratory studies of Hardy \& Bainbridge (1954) that showed that the vertical migratory responses of a large variety of zooplankton, including euphausiids, are triggered only by slowly changing light levels with no migratory behaviour occurring during conditions of total darkness imposed at any point in their diurnal cycle.

Multi-day fluctuations in total water-column scattering levels, for example the enhanced scattering around profile Hours 1000 to 1050 and the extended periods of depressed scattering between profile Hours 800 to 900 and Hours 1080 to 1120 observed in Figs. 1 \& 3a, may be due to advection of large-scale spatial concentration inhomogeneities through the region. The current structure revealed by the RDI current profiler was complex. After tidal components had been removed a fairly persistent westerly current drift of the order of $20 \mathrm{~cm} \mathrm{~s}^{-1}$ characterized the seasonally mixed layer while much-reduced currents occurred in the lower half of the water column with frequent episodes of deep eastward flowing counter currents. Our shipboard acoustic transects of the Scotian Shelf basins have shown considerable spatial variability in euphausiid scattering horizons on scale lengths of tens of kilometers. This suggests both the population spatial inhomogeneities and the systematic current fields necessary for advective variation are normally present but a detailed analysis is beyond the scope of the present paper.

The possibility of varying ambient light levels from cloud cover influencing the character of the migrations as evident in Fig. 1, such as slowing or weakening the diurnal transitions so that the euphausiids tend to linger at mid-water depths, was explored by examining time series of sky opacity for the nearest weather stations of Shearwater (Dartmouth) and Sable Island. No obvious correlations were evident. However these stations, especially Sable Island, are remote from the observation area in a geographic region where meteorological conditions vary on short spatial and temporal scales.

The upper water-column backscatterers observed prior to Hour 500 cannot be positively identified. If the scatterers were euphausiids their diurnal migratory behaviour was highly anomalous. We favour a transitory migratory fish population. Acoustic observations in adjacent Emerald Basin in November 1993 disclosed high populations of juvenile silver hake which displayed a diurnal migratory cycle confined to the upper water column. However, their observed daytime equilibrium depth below $70 \mathrm{~m}$ is deeper than the 30 to $40 \mathrm{~m}$ observed in the present data set. The inability to differentiate between fish or zooplankton scatterers illustrates the limitations of observing at a single acoustic frequency. Simultaneous observations at a second frequency preferably below $50 \mathrm{kHz}$ could easily decide the issue.

Little information exists on the orientational behaviour of Meganyctiphanes norvegica in their natural habitat. However, more study has been afforded the larger Antarctic species Euphausia superba which may be of value. Kils (1981) showed that during stationary hovering $E$. superba must be oriented about $55^{\circ}$ to the horizontal. However under small tank confinement some average forward motion is usually observed with an average orientation angle of about $45^{\circ}$ and standard deviation of about $30^{\circ}$. Because of hydrodynamic lift arising from their body shape a $E$. superba can move forward up to $0.13 \mathrm{~m} \mathrm{~s}^{-1}$ at constant depth without increasing metabolism above the hovering level. A minimum metabolic requirement occurs at $0.06 \mathrm{~m} \mathrm{~s}^{-1}$. The pleopods (propulsion appendages) are optimized for most efficient performance at about $0.15 \mathrm{~m} \mathrm{~s}^{-1}$. This fact suggest $E$. superba frequently utilize the higher velocity regions of their performance envelope. In situ diver observations (Hamner 1984) have revealed characteristic organized foraging activity with forward velocities up to $0.2 \mathrm{~m} \mathrm{~s}^{-1}$, comparable to the velocities for best pleopod efficiency. Kils' (1981) lab studies indicate a close relationship between forward swim speed and orientation. Forward velocities greater than 
$0.1 \mathrm{~m} \mathrm{~s}^{-1}$ for $E$. superba are associated with orientations of about $10^{\circ}$ to the horizontal. The overall body shape and hovering angle of the somewhat smaller $M$. norvegica are known to be similar to $E$. superba. Smaller size implies that for a given swimming speed drag resistance $\left(\approx L^{2}\right.$ dependence) will be proportionately more important than gravity ( $\approx L^{3}$ dependence), implying shallower swim angles versus forward velocity curves for $M$. norvegica. However, in isolation, this is not adequate to predict an average swim angle under natural conditions.

Kristensen \& Dalen (1986) made photographic observations of euphausiids (presumably Thysanoessa sp. and/or Meganyctiphanes norvegica) confined in large outdoor seawater pens. They found that the mean orientation (head and tail distinguished) varied from 'slightly positive to slightly negative during the day' with standard deviations between 25 and $45^{\circ}$. One published example showed a mean tilt of about $10^{\circ}$ at 02:00 h local time. Our previous photographic studies (Sameoto 1980) of $M$. norvegica and Thysanoessa raschii showed broad ill-defined orientation distributions during daytime not incompatible with standard deviations of the order of $30^{\circ}$. At night many observations showed a much more vertical orientation in the 45 to $90^{\circ}$ range. However since the effect of stray artificial light on orientation (Sameoto et al. 1985) was not appreciated at the time, the significance of the nighttime observations are in doubt. On the whole one might conservatively speculate that freely swimming $M$. norvegica might display an average orientation $\leq 15^{\circ}$ and a SD of about $30^{\circ}$. During upward migration it seems reasonable that the $55^{\circ}$ orientation angle for a purely upward-directed force vector would seldom be exceeded. Kils' (1981) observations that confined euphausids exhibit systematic forward motion would imply a behavioural preference for a lower orientational angle in order to maintain some horizontal movement. A $30^{\circ}$ average euphausiid orientation with a $30^{\circ} \mathrm{SD}$ would be compatible with our acoustic levels observed during upward migration without unduly diminishing the upward component of the swimming force vector. Kils (1981) also demonstrated that, while sinking, euphausiids, including $M$. norvegica, assume a virtually pure horizontal orientation and for a $0.028 \mathrm{~m}$ body length descend at about $0.022 \mathrm{~m} \mathrm{~s}^{-1}$. Since this agrees closely with our estimated downward migration velocity it is reasonable to believe the downward migration is achieved by sinking, a behaviour which would confer the additional value of conserving organism energy. A dominantly horizontal orientation while sinking would explain why integrated acoustic levels may drop slightly during ascent but not during descent. Note that for a near-horizontal orientation euphausiid target strength is virtually independent of the orientation angle SD (Table 4).
To summarize, it has been demonstrated that bottommounted sonars are useful for monitoring long-term fluctuations in biological abundances as well as delineating the mean behaviours of diurnally migrating biological horizons. Observations of these types are normally impractical using either acoustic or direct sampling from survey vessels. The utility of single acoustic frequency observations is critically dependent on unambiguously identifying the relevant backscattering horizons. This may restrict acoustic monitoring to regions of relatively well-known biology, or require a concurrent program of direct sampling. Many interpretational ambiguities could be eliminated by utilizing more than 1 acoustic frequency simultaneously.

Acknowledgements. We thank Don Lawrence, Coastal Oceanography Division, Bedford Institute of Oceanography, for supplying the Minimet temperature data and the Atmospheric Environment Service, Atlantic Region, Bedford, Nova Scotia for supplying detailed weather records for Shearwater and Sable Island. The officers and crew of CSS 'Dawson' are commended for their skill in handling the RDI bottom mount package. Valuable conversations and correspondence with T K. Stanton, Woods Hole Oceanographic Institution, are gratefully acknowledged. This project was in part funded by the Federal Panel on Energy Research and Development (PERD).

\section{LITERATURE CITED}

Chu, D., Foote, K. G., Stanton, T. K. (1993). Further analysis of target strength measurements of Antarctic krill at $38 \mathrm{kHz}$ and $120 \mathrm{kHz}$ : comparison with deformed cylinder model and inference of orientation distribution. J. Acoust. Soc Am. 93(5): 2985-2988

Clay, C. S., Medwin, H. (1977). Acoustical oceanography. John Wiley \& Sons, New York

Cochrane, N. A., Sameoto, D., Herman, A. W., Neilson, J. (1991). Multiple-frequency acoustic backscattering and zooplankton aggregations in the inner Scotian Shelf basins. Can. J. Fish. Aquat. Sci. 48: 340-355

Dessureault, J.-G., Belliveau, D. J., Young, S. W. (1991) Design and tests of a trawl-resistant package for an acoustic Doppler current profiler. IEEE J. Oceanic Eng. 16 $397-401$

Flagg, C. N., Smith, S. L. (1989). On the use of the acoustic Doppler current profiler to measure zooplankton abundance. Deep Sea Res. 36: 455-474

Foote, K. G., Everson, I., Watkins, J. L., Bone, D. G. (1990). Target strengths of Antarctic krill (Euphausia superba) at 38 and $120 \mathrm{kHz}$. J. Acoust. Soc. Am. 87(1): 16-24

Francois, R. E., Garrison, G. R. (1982a). Sound absorption based on ocean measurements. Part I: pure water and magnesium sulfate contributions. J. Acoust. Soc. Am. 72(3): $896-907$

Francois, R. E., Garrison, G. R. (1982b). Sound absorption based on ocean measurements. Part II: boric acid contribution and equation for total absorption. J Acoust. Soc. Am. 72(6): $1879-1890$ 
Greenlaw, C. F., Johnson, R. K. (1982). Physical and acoustical properties of zooplankton. J. Acoust. Soc. Am. 72(6): $1706-1710$

Hamner, W. M. (1984). Aspects of schooling in Euphausia superba. J. Crust. Biol. 4: 67-74

Hardy, A. C., Bainbridge, R. (1954). Experimental observations on the vertical migrations of planktonic animals. J. mar. biol. Ass. U.K. 33: 409-448

Herman, A. W., Cochrane, N. A., Sameoto, D. D. (1993). Detection and abundance estimation of euphausiids using an optical plankton counter. Mar. Ecol. Prog. Ser. 94: $165-173$

Herman, A. W., Sameoto, D. D., Chen Shunnian, Mitchell, M. R., Petrie, B., Cochrane, N. A. (1990). Sources of zooplankton on the Nova Scotia Shelf and their aggregations within deep shelf basins. Contin. Shelf Res. 11: $211-238$

Heywood, K. J., Scrope-Howe, S., Barton, E. D. (1991). Estimation of zooplankton abundance from shipborne ADCP backscatter. Deep Sea Res. 38; 677-691

Kils, U. (1981). The swimming behaviour, swimming performance and energy balance of Antarctic krill, Euphausia superba. BIOMASS Scientific Series No. 3

Koeller, P., Coates-Markle, L., Neilson, J. D. (1989). Feeding ecology of juvenile (0-group silver hake) Merluccius bilinearis on the Scotian Shelf. Can. J. Fish. Aquat. Sci. 46: $1762-1768$

Kristensen, A, Dalen, J. (1986). Acoustic estimation of size distribution and abundance of zooplankton. J. Acoust. Soc. Am. 80(2): 601-611

Meeus, J. (1988). Astronomical formulae for calculators. 4th edn. Willmann-Bell, Inc., Richmond, VA

RD Instruments. (1990). Calculating absolute backscatter. Technical Bulletin ADCP-90-04. Issued Dec. 1990. RD Instruments, San Diego, CA

This article was submitted to the editor
Roe, H. S. J., Griffiths, G. (1993). Biological information from an acoustic Doppler current profiler. Mar. Biol. 115: 339-346

Sameoto, D. D. (1980). Quantitative measurements of euphausiids using a $120-\mathrm{kHz}$ sounder and their in situ orientation. Can. J. Fish. Aquat. Sci. 37: 693-702

Sameoto, D., Cochrane, N. A., Herman, A. W. (1985). Response of biological backscattering to ship's lights. Can. J. Fish. Aquat. Sci. 42: 1535-1543

Sameoto, D., Cochrane, N., Herman, A. (1993). Convergence of acoustic, optical, and net-catch estimates of euphausiid abundance: the use of artificial light to reduce net avoidance. Can. J. Fish. Aquat. Sci. 50: $334-346$

Sameoto, D. D., Herman, A. W. (1990). Life cycle and distribution of Calanus finmarchicus in deep basins on the Nova Scotia shelf and seasonal changes in Calanus spp. Mar. Ecol. Prog. Ser. 66: 225-237

Sameoto, D. D., Jaroszynski, L. O., Fraser, W. B. (1980) BIONESS: a new design in multiple net zooplankton samplers. Can. J. Fish. Aquat. Sci. 37: 722-724

Stanton, T. K. (1989). Simple approximate formulas for backscattering of sound by spherical and elongated objects. J. Acoust. Soc. Am. 86(4): 1499-1510

Stanton, T K., Chu, D., Wiebe, P. H., Clay, C. S. (1993a) Average echoes from randomly-oriented random-length finite cylinders: zooplankton models. J. Acoust. Soc. Am 94(6): $3463-3472$

Stanton, T. K., Clay, C. S., Chu, D. (1993b). Ray representation of sound scattering by weakly scattering deformed fluid cylinders: simple physics and application to zooplankton J. Acoust. Soc. Am. $94(6): 3454-3462$

Thomson, R. E., Gordon, R. L., Dymond, J. (1989). Acoustic Doppler current profiler observations of a mid-ocean ridge hydrothermal plume. J. geophys. Res. 94: 4709-4720

Thorpe, S. A. (1992). Bubble clouds and the dynamics of the upper ocean. Q. J. R. Meteorol. Soc. 118: 1-22

Manuscript first received: September 29, 1993

Revised version accepted: January 11, 1994 